Shrestha, S. M. B., Joldes, G. R., Wittek, A. and Miller, K. (2013), Cellular automata coupled with steady-state nutrient solution permit simulation of large-scale growth of tumours. Int. J. Numer. Meth. Biomed. Engng., 29: 542-559. doi: 10.1002/cnm.2539

(c) 2013 John Wiley \& Sons, Ltd.

This is the peer reviewed version of the following article Shrestha, S. M. B., Joldes, G. R., Wittek, A. and Miller, K. (2013), Cellular automata coupled with steady-state nutrient solution permit simulation of large-scale growth of tumours. Int. J. Numer. Meth.

Biomed. Engng., 29: 542-559. doi: 10.1002/cnm.2539, which has been published in final form at http://dx.doi.org/10.1002/cnm.2539. This article may be used for noncommercial purposes in accordance with Wiley Terms and Conditions for self-archiving.

This version was made available in the UWA Research Repository on 1 April 2014 in compliance with the publisher's policies on archiving in institutional repositories.

Use of the article is subject to copyright law. 


\title{
Cellular Automata Coupled with Steady-State Nutrient Solution Permit Simulation of Large-Scale Growth of Tumours
}

\author{
Sachin Man Bajimaya Shrestha, Grand Roman Joldes, Adam Wittek and Karol Miller \\ Intelligent Systems for Medicine Laboratory \\ The University of Western Australia, 35 Stirling Highway, Crawley WA 6009, Australia \\ Email: kmiller@mech.uwa.edu.au
}

\begin{abstract}
SUMMARY
We model complete growth of an avascular tumour by employing cellular automata for the growth of cells and steady-state equation to solve for nutrient concentrations. Our modelling and computer simulation results show that, in the case of a brain tumour, oxygen distribution in the tumour volume may be sufficiently described by a time-independent steady-state equation without losing the characteristics of a time-dependent diffusion equation. This makes the solution of oxygen concentration in the tumour volume computationally more efficient thus enabling simulation of tumour growth on a large scale. We solve this steady-state equation using central difference method. We take into account the composition of cells and inter-cellular adhesion in addition to processes involved in cell cycle - proliferation, quiescence, apoptosis and necrosis - in the tumour model. More importantly, we consider cell mutation that gives rise to different phenotypes and therefore, a tumour with heterogeneous population of cells. A new phenotype is probabilistically chosen and has the ability to survive at lower levels of nutrient concentration and reproduce faster. We show that heterogeneity of cells that compose a tumour leads to its irregular growth and that avascular growth is not supported for tumours of diameter above $18 \mathrm{~mm}$. We compare results from our growth simulation with existing experimental data on Ehrlich ascites carcinoma and tumour spheroid cultures, and show that our results are in good agreement with the experimental findings.
\end{abstract}

KEY WORDS: tumour growth, heterogeneous tumour, phenotypical evolution, oxygen concentration, hybrid computation, cellular automata 


\section{INTRODUCTION}

Cancer is a disease that accounts for more than one-fifth of all deaths in industrialised countries of the Western World. Likewise, one person out of three will be treated for a severe cancer in their lifetime [1]. In less-industrialised countries, they often manifest at younger ages than in industrialised countries. Unfortunately, overall, neither incidence nor mortality of human cancer has been much diminished by conscious human intervention over the last decades. It is hoped that a better understanding of the cellular basis underlying tumour growth will eventually open the door to its successful treatment, as will the development of novel drugs and therapies based on the results of molecular and cellular biological cancer research.

Tumour growth is a multistage process. Mutations in a single normal cell lead to loss of its homeostatic mechanism which is the fundamental regulatory mechanism of cells. This leads to inappropriate mitosis (cell division) and loss of apoptosis, a process by which cells die after exceeding their natural lifespan [2]. The normal cell thus transforms to a cancerous cell. The cell proliferates unregulated and gives rise to a heterogeneous irregular tumour growth. The size of initial growth is dependent on the supply of nutrients, in particular, oxygen, through diffusion [3] and this initial phase is called the avascular growth phase. Once tumour reaches the diffusion-limited size, it has to recruit blood vessels to supply it with further nutrients. The tumour does so in the second phase through angiogenesis. The new vessels enhance the supply of nutrients allowing the tumour to enter the vascular phase. At this stage, tumour cells proliferate aggressively and metastasize thus invading the surrounding tissue. The characteristic properties that define cancer thus include uncontrolled cell proliferation, altered differentiation and metabolism, genomic instability, and invasiveness with eventual metastasis.

The avascular phase is also called the primary growth phase and is considered relatively benign. The detection and treatment of tumour at this stage has a greater probability of having the disease cured. On the other hand, the vascular phase, also called the secondary growth phase, is more malignant and treatment becomes far more difficult at this stage, on most occasions leading to serious complications.

In this paper, we model tumour growth in the avascular phase. The size and shape of a tumour at this stage is predominantly determined by its cellular composition, time required for cell division (mitosis), cell mutation (phenotypical evolution), intercellular adhesion, 
concentration of vital nutrients, and mechanical stresses from surrounding tissue, for example, in the case of a brain tumour, mechanical stresses due to confinement in the skull. Most existing models either avoid solving for nutrient concentration or simulate growth on a rather small size of lattice $[4,5]$. While the former is due to the inherent time-consuming nature of numerical solution to time-dependent partial differential equations, the latter is because simulating growth on a macro-scale starting from a few cells using cellular automata is computationally very expensive. Therefore, a majority of the research in the area are limited to pattern formation in a growing tumour $[4,6]$ rather than in its complete growth. In this work, we propose and demonstrate that a pure cellular automata growth model coupled with a steady-state solution to nutrient concentration, in the case of brain tumour, can be used to simulate complete growth.

Tumour heterogeneity contributes to its irregular shape. A tumour mass consists of three types of cells - proliferating, quiescent and necrotic. Cells, mostly on the tumour boundary, that are exposed to high levels of oxygen concentration undergo cell division and lead to tumour proliferation. In contrast, cells at the centre of tumour suffocate due to lack of oxygen and die (necrosis) forming a necrotic core. Moreover, some cells die after naturally exceeding their lifespan (apoptosis) and are seen scattered in the tumour mass. Some cells in the mass are exposed to nutrient levels that are higher than suffocation levels but insufficient to promote proliferation. Such cells are dormant and are called quiescent cells. They neither die nor undergo cell division. However, they participate in the normal cell cycle once sufficient level of oxygen is restored. In addition, some tumour cells mutate and give rise to a different phenotype that survive at smaller nutrient concentrations and proliferate faster. This heterogeneous population of cells leads to different velocities of growth in different directions forming an asymmetric irregular tumour volume.

To date, tumour growth modelling approaches include the continuum [7-14], discrete, and hybrid continuum-discrete approaches [15-18]. Continuum models are based on balance laws - balance of mass of the several components of tissue, balance of momentum and balance of energy - for the description of cell population [11] while a set of reaction diffusion equations are devised for nutrients and chemicals that influence growth. However, growth description through such modelling is phenomenological and it does not reflect the microscopic mechanisms of cancerous growth, such as proliferation, necrosis and apoptosis as well as the mechanical pressure inside tumour. Continuum models, therefore, are not sensitive to small fluctuations in the tumour growth system. This is a significant shortcoming as in some cases 
such small changes can be the leading cause in driving a nonlinear complex bio-system to a different state. Continuum approaches have however been successfully used in modelling tissues on a macroscopic scale $[19,20]$ primarily because cell level modelling for such a size is limited due to existing computational power.

Discrete models, on the other hand, can represent individual cells in space and time and can incorporate biological rules to define behaviour at the level of cells. Such models better respond to small changes in the tumour system. In this paper, we make use of a hybrid discrete-continuum approach in a bid to take advantage of the strengths of both of these approaches. In particular, we solve partial differential equations for the oxygen concentrations in the tissue while cellular automata (CA) are used to model tumour growth at the cell level. CA is a collection of cells on a grid of specified shape that synchronously evolves through a number of discrete time steps, according to an identical set of rules applied to each cell based on the states of its neighbouring cells [21, 22]. The grid can be implemented in any finite number of dimensions, and neighbours are a selection of cells relative to a given cell.

Mathematical modelling of tumour growth dates back to as early as 1972 when Greenspan [6] modelled simple tumour growth by diffusion to study growth characteristics from the most easily obtained data, i.e., growth in terms of movement of the outer radius of tumour as a function of time. His study concentrated on the steady-state histology with an objective to infer the major internal processes that affected tumour growth and observed that growth retardation is an effect of the formation of a necrotic core. Other studies that employ continuum models include reaction diffusion model by Gatenby et al. [8] to describe spatial distribution and temporal development of tumour tissue. Ward et el. [9] modelled avascular tumour by using non-linear partial differential equations that took into account only two types of cells - cancer and dead. Ferreira et al. [10] extended the reaction diffusion model by including cell motility in their model. Ambrosi et al. [11] described growth as an increase of the mass of the particles of the body and not as an increase of their number and modelled growth using continuum mechanics framework. Later, Byrne et al. [12] built their model using the theory of mixtures and Cristini et al. [13] performed nonlinear simulations of tumour using the mixture model. An earlier review on mathematical modelling of tumour is by Araujo et al. [14].

Cellular automata modelling of tumour growth is relatively young compared to continuum modelling. One of the early cellular automata models of tumour was developed by Qi et al. 
[4]. They modelled tumour growth using two-dimensional cellular automata on a very small grid-size. Immune system surveillance against cancer was taken into account. The model was based on the assumptions that cell division occurs only in the presence of an empty space in one of its nearest neighbours and that dead cells dissolve and disappear instead of forming a necrotic core as seen in in vivo tumours. Kansal et al. [23] modelled growth to reproduce the macroscopic structure of a tumour arising from microscopic processes. However, the transition rules used in the model are neither local nor homogeneous and therefore deviate from the core definition of cellular automata. Moreover, the nutrient gradient is always considered originating from the centre of the tumour mass and directed outwards toward the tumour boundary. This does not resemble a biological growth situation since the necrotic core, which is a mass of dead cells, does not consume nutrients. If nutrients are not consumed whilst still diffusing, the computed gradient lasts for only a very short period after cells have become necrotic. Dormann et al. [5] employed lattice-gas cellular automata (LGCA) to model self-organised avascular tumour. Although LGCA is extensively used in fluid models, we propose that growth modelled purely using cellular automata at the level of a single cell will be more representative of in vivo tumour growth. Their model was simulated in a $200 \times 200$ grid, a relatively small lattice, starting from 44 cancer cells, a fairly large initial number of cells. Moreover, their model does not include the phenotypical evolution - presence of mutated, more aggressive cancer cells - of tumour. Study by Vermeulen et al. [6] on tumour spheroid cultures provides evidence that a single cancer cell can self-renew and reconstitute a complete and differentiated carcinoma thus making the tumour population heterogeneous. As tumour grows, proliferating cancer cells are thus seen to give rise to more aggressive phenotypes different from the parent cell. Anderson [16] used a hybrid discrete-continuum model to examine the effect of cell-cell and cell-matrix adhesion upon the invasion of healthy tissue by a growing tumour. Specifically, the model considers early vascular growth just after angiogenesis has occurred and so, focuses on the secondary growth of tumour. Alarcon et al. [17] made use of hybrid cellular automata as a basic theoretical framework to model tumour at a multi-scale wherein intercellular processes are represented by ordinary differential equations and extracellular processes by partial differential equations. Gevertz et. al. [24] employed cellular automata to couple vascularisation with cellular growth in tumour. The study thus focused on the angiogenetic growth. Gerlee et. al. [25] built a model for tumour growth by employing cellular automata together with artificial neural network. Although 
oxygen concentration is not explicitly solved for in their model, they conclude that tissue background oxygen concentration affects the dynamics of tumour growth.

Wise et al. [26] provided a numerical algorithm for continuum modelling of diffuseinterface in multispecies tumour growth. Most recently in [27], they extended the continuum diffuse-interface method by incorporating hybrid-discrete continuum method for cell movement to model tumour-induced angiogenesis. Their latter work, directed on the secondary growth of tumour, concluded that invasion may be a function of heterogeneity. Sottoriva et al. [18] implemented the cancer stem cell concept to explain invasive tumour morphology using the hierarchical organisation of cell species. Their model incorporates the phenotypical evolution of cancer cells. However, despite solving for oxygen concentration to decide on the fate of cells, their model assume that cells die if they are at a depth of 60 cells or greater from the tumour boundary.

In the work presented here, we propose a number of improvements over the methods previously presented in the literature. We build upon the CA model from our previous work [28] where we showed that the heterogeneity of cells that compose a tumour leads to its irregular growth. We incorporate the composition of cells and inter-cellular adhesion in addition to processes involved in cell cycle - proliferation, quiescence, apoptosis and necrosis - in our tumour growth model. More importantly, we consider cell mutation that gives rise to a different phenotype and therefore, a tumour with heterogeneous population of cells. A new phenotype is probabilistically chosen and has the ability to survive at lower levels of nutrient concentration and reproduce faster. The fate of cells in our model is determined by the availability of nutrients whose concentration is described by a partial differential equation. We use our method to simulate a complete avascular growth of tumour which, to our knowledge, has not been done so far. Our method not only enables simulation of tumour growth on a large scale but also permits a more computationally efficient growth simulation and so, the growth simulation is faster. The scheme for this study is depicted in Fig.1.

\section{THE CELLULAR AUTOMATA TUMOUR GROWTH MODEL}

We represent tumour by a discrete set of cells on a two dimensional lattice $\Omega$ of $\mathrm{N} \times$ $\mathrm{N}$ cells with zero-flux boundary conditions. Such a boundary condition is chosen so that the cancer cells do not proliferate outside the brain tissue. We choose the Von Neumann neighbourhood. As shown in Fig.2.a, the neighbourhood consists of the cell in consideration 
and four other cells at length of one cell on its right, left, top and bottom. A cellular automaton is a point in the lattice representing a cell which can be in a proliferating, quiescent or necrotic (dead) state. The size of a cell is $10 \mu \mathrm{m} \times 10 \mu \mathrm{m}$ [29]. Automaton rules will be given in Section 4.

\section{Possible location of Figure 1.}

Fig.1. The scheme of study.

\section{Possible location of Figure 2.}

Fig.2. (a) The von Neumann neighbourhood consists of cells at positions $(0,0),( \pm 1,0),(0, \pm 1)$, i.e., the four yellow cells and the cell C. (b) A daughter cell can take position at site 1, 2, 3 or 4 with equal probability. However, the cell existing at that site has to be displaced to one of its neighbouring blue sites first to create space for the daughter cell.

\section{SOLUTION TO THE OXYGEN DIFFUSION EQUATION}

The oxygen distribution in the tumour volume and its immediate surroundings during the growth process is governed by the reaction-diffusion equation

$$
\frac{\partial \mathrm{c}(\mathbf{r}, \mathrm{t})}{\partial \mathrm{t}}=\mathcal{D} \nabla^{2} \mathrm{c}(\mathbf{r}, \mathrm{t})-\mathrm{k}_{\mathrm{i}}(\mathbf{r}) \quad \mathrm{k}_{\mathrm{i}} \in\left(\mathrm{k}_{\mathrm{p}_{1}}, \mathrm{k}_{\mathrm{p}_{2}}, \mathrm{k}_{\mathrm{p}_{3}}, \mathrm{k}_{\mathrm{p}_{4}}, \mathrm{k}_{\mathrm{q}}\right)
$$

where, $\mathcal{D}=1 \times 10^{-5} \mathrm{~cm}^{2} \mathrm{~s}^{-1}[13]$, is the coefficient of diffusion; $\mathrm{c}(\mathbf{r}, \mathrm{t})$ is the magnitude of oxygen concentration at the cellular automaton (CA) element at location $\mathbf{r}$ at time $\mathrm{t}$; $\mathrm{k}(\mathbf{r})$ is the rate of oxygen consumption by the CA element at $\mathbf{r}$ and is dependent on the type of cell, $\mathrm{k}_{\mathrm{i}}$. If we choose the number of phenotypes to which a proliferating cancer cell can mutate or differentiate to be four, $\mathrm{k}_{\mathrm{i}}=\mathrm{k}_{\mathrm{p}_{\mathrm{j}}}, \mathrm{j}=1,2,3,4$ for proliferating cells of phenotype I, II, III and IV respectively, and $\mathrm{k}_{\mathrm{i}}=\mathrm{k}_{\mathrm{q}}$ for quiescent cells. 
In two dimensions, Eq.1 may be written as

$$
\begin{aligned}
& \frac{\partial \mathrm{c}(\mathbf{x}, \mathbf{y}, \mathrm{t})}{\partial \mathrm{t}}=\mathcal{D}\left(\frac{\partial^{2} \mathrm{c}(\mathbf{x}, \mathbf{y}, \mathrm{t})}{\partial \mathrm{x}^{2}}+\frac{\partial^{2} \mathrm{c}(\mathbf{x}, \mathbf{y}, \mathrm{t})}{\partial \mathrm{y}^{2}}\right)-\mathrm{k}_{\mathrm{i}}(\mathbf{x}, \mathbf{y}), \\
& r_{\text {left }}<\mathrm{x}<r_{\text {right }}, \quad r_{\text {top }}<\mathrm{y}<r_{\text {bottom }}
\end{aligned}
$$

subject to the boundary condition [30]

$$
\begin{aligned}
& c\left(r_{\text {left }}, y, t\right)=c\left(r_{\text {right }}, y, t\right)=O_{h}=1 \times 10^{-4} \mathrm{gcm}^{-3}, \quad r_{\text {top }}<\mathrm{y}<r_{\text {bottom }}, \quad t>0, \\
& c\left(x, r_{\text {top }}, t\right)=c\left(x, r_{\text {botttom }}, t\right)=O_{h}=1 \times 10^{-4} \mathrm{gcm}^{-3}, \quad r_{\text {left }}<\mathrm{x}<r_{\text {right }}, \quad t>0,
\end{aligned}
$$

and initial condition

$c(x, y, 0)=k_{p}=10^{-6} \mathrm{gcm}^{-3}[18,30], \quad r_{\text {left }}<\mathrm{x}<r_{\text {right }}, \quad r_{\text {top }}<\mathrm{y}<r_{\text {bottom }}$.

Here, $r_{\text {dir }}$, dir $\in$ (left, right, top, bottom) is the maximum radius of tumour in the left and right $\mathrm{x}$-directions and, top and bottom y-directions at time, $t . O_{h}[30]$ is the magnitude of oxygen concentration in the healthy brain tissue. $k_{p}[18,31]$ is the proliferating oxygen concentration threshold. We choose the initial concentration of oxygen inside the tumour lattice to be equal to $k_{p}$ so that the initially seeded cancer cells in the tumour are all in the proliferating state. We then solve for oxygen concentration in a spherical volume. The concentration outside the volume is considered to be equal to the oxygen concentration in a normal tissue. Therefore, in this part of the model artificial anisotropy is avoided.

Eq.2 may be solved by either an implicit or an explicit method. Here, we choose the explicit method for reasons explained shortly. In explicit numerical method, to achieve a stable solution to Eq.2, the condition $0<\lambda \leq 1 / 2$ has to be met where, $\lambda=\mathcal{D}\left(k / h^{2}\right)[32-$ 34].

Here, $\lambda$ is the mesh-ratio parameter, $k$ is the space-step size and $h$ is the time-step size. To solve for oxygen concentration at each site of a cell, we take the space-step size to be equal to the size of a cell. For such a space-step size the magnitude of time-step that can be used from the above equation even when $\lambda=1 / 2$ - the maximum possible value for $\lambda$ - is very small and is in the order of a hundredth of a second. The time required for a mitotic cycle being about 16 hours [30], the equation has to be solved about 1.5 million times for every mitotic cycle and is therefore computationally very expensive.

Our experience [28, 33, 34] in addition to existing evidence [32] shows that implicit methods are intrinsically computationally very intensive too and have no advantage over 
explicit methods in terms of speed at such small space-step size and very large total time for which the equation has to be solved. Therefore, we solve Eq.2 using the explicit numerical method.

We find that in the case of brain tissue, both the rate of diffusivity, compared to that of other tissues such as the bone, and the mitotic cycle time - 16 hours - are very high. Therefore, we hypothesise that the oxygen distribution in the brain tissue reaches a steadystate within a mitotic period. First, we justify this hypothesis by showing that, in the case of brain tissue, the time-independent steady-state equation can sufficiently describe the distribution of oxygen in the tumour lattice without losing the characteristics of the timedependent diffusion equation (Eq.2). In the case of a growing tumour, we will initially be interested in finding the distribution of oxygen in the lattice before it is consumed by cells. This initial distribution prior to consumption is given by the equation

$$
\frac{\partial \mathrm{c}(\mathbf{x}, \mathbf{y}, \mathrm{t})}{\partial \mathrm{t}}=\mathcal{D}\left(\frac{\partial^{2} \mathrm{c}(\mathbf{x}, \mathbf{y}, \mathrm{t})}{\partial \mathrm{x}^{2}}+\frac{\partial^{2} \mathrm{c}(\mathbf{x}, \mathbf{y}, \mathrm{t})}{\partial \mathrm{y}^{2}}\right)
$$

The oxygen is consumed by the cells that compose the tumour volume during a mitotic period, the time in which proliferation occurs. However, once proliferation has taken place, a significantly high amount of time (16 hours) has to elapse before another proliferation step takes place. Therefore, for small concentrations of oxygen as seen in tumour lattice, the left hand side of Eq.3 tends to zero for such a long time period. This leads to the following steady-state equation

$$
\left(\frac{\partial^{2} \mathrm{c}(\mathbf{x}, \mathbf{y})}{\partial \mathrm{x}^{2}}+\frac{\partial^{2} \mathrm{c}(\mathbf{x}, \mathbf{y})}{\partial \mathrm{y}^{2}}\right)_{\mathrm{t}}=0, \quad r_{\text {left }}<\mathrm{x}<r_{\text {right }}, \quad r_{\text {top }}<\mathrm{y}<r_{\text {bottom }}
$$

which is subject to the boundary condition

$$
\begin{array}{ll}
c\left(r_{\text {left }}, y\right)=c\left(r_{\text {right }}, y\right)=O_{h}=1 \times 10^{-4} \mathrm{gcm}^{-3}, & r_{\text {top }}<\mathrm{y}<r_{\text {bottom }}, \\
c\left(x, r_{\text {top }}\right)=c\left(x, r_{\text {botttom }}\right)=O_{h}=1 \times 10^{-4} \mathrm{gcm}^{-3}, & r_{\text {left }}<\mathrm{x}<r_{\text {right }} .
\end{array}
$$

Here, $t$ is an integer multiple of mitotic period. Eq.2, which is a boundary and initial value problem, thus reduces to a boundary value only problem represented by Eq.4. Eq.4 may be used to solve for the concentration of oxygen in the tumour volume after every mitotic period. After consumption by cells, the concentration of oxygen in the tumour lattice is given by the equation 


$$
\left(\frac{\partial^{2} \mathrm{c}(\mathbf{x}, \mathbf{y})}{\partial \mathrm{x}^{2}}+\frac{\partial^{2} \mathrm{c}(\mathbf{x}, \mathbf{y})}{\partial \mathrm{y}^{2}}\right)_{\mathrm{t}}-\mathrm{k}_{\mathrm{i}}(\mathbf{x}, \mathbf{y})_{\mathrm{t}}=R_{O_{2}}
$$

where, $R_{O_{2}}<0$ means that the cells will become necrotic. $R_{O_{2}} \geq 0$ is the residual amount of oxygen left after consumption by cells. When $R_{O_{2}}>0$, the residual oxygen is available for further diffusion.

Next, to further strengthen our confidence in this hypothesis, we compared the oxygen distribution in various sizes of brain tumour lattices by solving both the time-dependent (parabolic) diffusion equation and the time-independent steady-state (elliptic) equation, in this case, the Laplace equation. Fig. 3 shows the plots for lattice sizes $200 \times 200$ cells (Fig.3.a, 3.b), $400 \times 400$ cells (Fig.3.c, 3.d) and $2000 \times 2000$ cells (Fig.3.e, 3.f) wide. The size of the lattices was fixed while solving for oxygen concentration meaning the equations are solved for a fixed size of tumour. Interestingly, we observed that the pattern and magnitude of oxygen distribution in the tumour volume were very similar in both the timeindependent and the time-dependent cases. Although the difference in the magnitude of concentration increased and peaked between 40 to 60 cells from the tumour boundary to a maximum of about $14 \%$, it sharply dropped to less than $3 \%$ at a depth of a hundred cells for tumours of radius greater than 100 cells. In both cases, the concentration reached near zero levels at about 100 to 120 cells from the tumour boundary.

\section{Possible location of Figure 3.}

Fig.3. (a), (c), (e) Oxygen profiles (concentrations in cell $^{-1}$ ) using steady-state timeindependent (Laplace) and time-dependent equations for fixed lattice sizes of $200 \times 200$, $400 \times 400$, and $2000 \times 2000$ cells respectively. Profiles are shown from the tumour boundary to its centre. (b), (d), (f) The difference in oxygen concentration corresponding to the three lattice sizes respectively. 
Further, we also tested this hypothesis against varying sizes of tumour lattice by solving both the time-dependent as well as time-independent equations on a growing tumour for increasing times. We chose to increase the size of tumour lattice from 3 cells $\times 3$ cells to 240 cells $\times 240$ cells with a corresponding increase in the number of mitotic periods to capture the growth of in vivo tumour.

Fig. 4 shows the oxygen profiles from the tumour boundary to its centre for blocks of time. In this study, we chose to compare the oxygen profile in the growing tumour after every 10 mitotic cycles and hence, each block, $x$, is equal to 10 mitotic cycles. Time blocks, shown with vertical lines, in the plots, except the first block, are multiples of $x$. The size of tumour corresponds to the size obtained while growing during the blocks of mitotic periods. We observed that the oxygen profiles in the case of the time-independent solution (Fig.4.a) are similar to those of the time-dependent solution (Fig.4.b) and that they follow analogous trends. Concentrations obtained through the solutions to the time-independent steady-state equation (Fig.4.c) and the time-dependent diffusion equation (Fig.4.d) for each of the mitotic blocks differed from as little as $1 \%$ for smaller sizes of tumour like 100 cells in diameter to at the most $6 \%$ for larger sizes of tumour like 240 cells in diameter (Fig.4.e). It is worth noting here that the maximum of the differences occurs at the tumour core and this difference will continue to increase for larger diameters of tumour. This will not, however, affect the growth dynamics since the core consists of necrotic cells that do not consume oxygen. For all lattice sizes, the difference between the oxygen concentration obtained through the steady-state solution and that obtained through the time-dependent solution is minimal (about 1\%) where it matters the most i.e., towards the tumour boundary. Therefore, the viable cells in both cases are exposed to similar amounts of oxygen.

Hence, we choose to use the time-independent equation (Eq.4) for solving the oxygen concentration in our model. While the time-dependent equation requied about $5.33 \mathrm{hrs}$ to solve for oxygen concentration in a growing lattice size that grew to a maximum of $120 \times$ 120 cells, the time-independent solution to the growing lattice of the same size took only about 6 minutes in a $3.2 \mathrm{GHz}$ PC with 4GB of RAM. 


\section{Possible location of Figure 4.}

Fig.4. Oxygen profiles (concentrations in cell $^{-1}$ ) using (a) time-independent and (b) timedependent diffusion equations for varying lattice sizes. The right edge of each plot (120 cells) represents the centre of tumour volume. Profiles at various blocks of time with their corresponding sizes of lattice using (c) time-independent and (d) time-dependent equations for varying lattice sizes. The left edge of each plot represents the boundary of the tumour volume. (e) Difference in the oxygen concentration between the steady-state timeindependent and time-dependent solutions for different tumour diameters (in number of cells).

We solve Eq.5 using the central difference method to determine the oxygen concentration at each node in the lattice. We use the Dirichlet boundary condition with the oxygen concentration at the boundary being constant and equal at all times to the oxygen concentration level of a healthy tissue at $1 \times 10^{-4} \mathrm{gcm}^{-3}[30]$; i.e., $\mathrm{c}\left(r_{\text {left }}, y\right)=$ $\mathrm{c}\left(r_{\text {right }}, y\right)=\mathrm{c}\left(x, r_{\text {top }}\right)=\mathrm{c}\left(x, r_{\text {botttom }}\right)=1 \times 10^{-4} \mathrm{gcm}^{-3}$. For simulation purposes, we convert the oxygen diffusivity in brain tissue as well as the rates of consumption by cells to the unit of a cell (Table I). To further save computation time, we solve the diffusion equation after every 10 mitotic cycle times. Our experience through previous work [28, 33, 34] indicate that it is sufficient to solve the diffusion equation after every 10 mitotic cycle times to obtain a desirable solution whereas solving the equation after larger steps of mitotic cycle times such as 20 or more has a smoothing effect on the oxygen profile and hence, is undesirable.

Table I. Variables used in the CA Model

\begin{tabular}{lcl}
\hline \multicolumn{1}{c}{ Variable } & Symbol & \multicolumn{1}{c}{ Value } \\
\hline $\mathrm{O}_{2}$ diffusion coefficient & $D$ & $10 \mathrm{cell} \mathrm{s}^{-1}$ \\
$\mathrm{O}_{2}$ concentration of healthy tissue & $O_{h}$ & $10^{-13} \mathrm{~g} \mathrm{cell}^{-1}$ \\
$\mathrm{O}_{2}$ consumption rate of proliferative cells & $k_{p}$ & $10^{-15} \mathrm{~g} \mathrm{cell}^{-1} \mathrm{~s}^{-1}$ \\
$\mathrm{O}_{2}$ consumption rate of quiescent cells & $k_{q}$ & $5 \times 10^{-16} \mathrm{~g} \mathrm{cell}^{-1} \mathrm{~s}^{-1}$ \\
\hline
\end{tabular}


Our model describes the avascular tumour growth wherein the size of the tumour is relatively small and so, has a very small microenvironment in contact with it. Therefore, it is sufficient to assume a homogeneous soft microenvironment for the growing tumour. For larger sizes of tumour, the effect of pressure on its growth may be incorporated by following our earlier work [35].

\section{AUTOMATON RULES}

1. The state of a CA element determines the type of cell in that element. The applied CA rules depend on the type of cell.

2. If an automaton element is a cancer cell, it can divide into daughter cells if all of the following is true.

(a) The level of oxygen concentration $C^{t}(x, y)$ at the position of that element is equal or greater than the proliferative threshold, $k_{p}=1 \times 10^{-6} \mathrm{gcm}^{-3}[18,31]$, implying that proliferation of a cancer cell occurs only when the amount of oxygen available to the cell is enough to let it do so.

(b) We assume that if a cancer cell is completely surrounded by cancer cells only, the cell will not have enough nutrients such as glucose to permit it to undergo cell division despite the availability of oxygen due to the effect of 'crowding'. Therefore, at least one normal cell should exist either to the right, left, top or bottom of the cell for the cancer cell to divide into daughter cells $[5,18]$. If this is the case, the normal cell is pushed to one of the normal cell's neighbourhood and the cancer cell proliferates into this vacant space.

(c) The age of the cell has not exceeded its lifespan. Cells have their biological lifespan. We allow cells to reproduce until five generations $[6,15,28]$. At this point, if the cell division does not lead to mutated daughter cells, the cell will die naturally.

When (a), (b) and (c) are all true, tumour growth is permitted. An empty place for the daughter cell is created in one of its neighbouring sites by displacing cells in the surrounding outwards. However, the position in which the daughter cell will move into would not be known yet and therefore, is evaluated first. If cell $\mathrm{C}$ can reproduce, the daughter cell can take at random one of four positions 1, 2, 3 or 4 (yellow sites in Fig.2.b) with equal probability. Once the position the daughter cell is going to occupy is determined, the normal cell occupying that position is pushed to one of the sites in the 
neighbourhood of the normal cell (blue sites in Fig.2.b) with equal probability. Therefore, growth is a result of two processes - propagation of a normal cell into its neighbourhood to create a space for the daughter cell followed by the proliferation of cancer cell into this vacant position from where a normal cell was displaced.

Recent models $[4,23,36]$ assume presence of at least one pre-existing empty space in the neighbourhood of a CA element (to be occupied by a daughter cell) as a necessary condition for a proliferating cell to divide. While simplifying the modelling process, this is not an accurate description of the way in which proliferation occurs. Rather, biologically, an empty space is created followed by proliferation of a daughter cell into this space [1]. This is accounted for in our model as described in 2 above.

3. If the level of oxygen concentration $C^{t}(x, y)$ falls below the proliferative threshold value $k_{p}=1 \times 10^{-6} \mathrm{gcm}^{-3}$, but is greater than the necrotic threshold value $k_{n}=k_{q}=$ $5 \times 10-7 \mathrm{gcm}-3[18,37]$ and the state of the automaton element is proliferating, the state changes to the quiescent state.

4. If the level of oxygen concentration $C^{t}(x, y)$ falls below $k_{q}$, the state of an automaton element in both the proliferating as well as the quiescent state changes to the necrotic state.

5. In the case of an automaton element that is in the quiescent state, if the local level of oxygen concentration is restored to or above the proliferative threshold $k_{p}$, it changes to the proliferating state.

6. We model intercellular adhesion by considering the number of similar external automaton elements a cell is attached to, $\mathrm{Q}$ [16]. If $\mathrm{Q} \geq 2$, a cell adheres to its neighbours whereas if $\mathrm{Q}_{1}<2$, the cell is allowed to migrate.

7. Cancer cells differentiate as they reproduce $[1,6]$. This gives rise to a cell of a phenotype different from the parent cell. The new phenotypes, in general, have more aggressive proliferating capability, meaning, they can survive and reproduce at smaller levels of oxygen concentrations than that required by their parent cell. Similarly, when these daughter cells mature and reproduce, they may mutate to cells of yet another phenotype that can proliferate even more aggressively, thus requiring even less oxygen to survive and reproduce. To model mutation, we consider four different phenotypes. Initially, all cells are of phenotype I. A cell can mutate with a probability $\mathrm{P} \_$mut $=0.1$ to one of phenotypes II, III or IV. Although the value of $\mathrm{P}_{-}$mut as 0.1 is an arbitrary first approximation, this 
value corresponds to the fraction of mutated or differentiated cancer cells observed in various solid malignancies [6]. Phenotype II can proliferate at half the nutrient concentration required by phenotype I and can reproduce at half the time required by phenotype I. We proceed similarly to determine the time and oxygen required by phenotype III and IV to proliferate. Anderson [16] used, in addition to the above, a random mutation sequence where all the cells are initially assigned to one of 100 phenotypes randomly and through mutation, another phenotype is selected randomly. He concluded that whilst these two methods for considering tumour cell heterogeneity are different, they ultimately produce similar results.

8. When the age of a cell equals its lifespan, we check if it can mutate. If the cell can mutate, it can acquire a different phenotype as mentioned in 7 above. The age of the cell is then reset to zero. Otherwise the cell will die due to exceeding its lifespan naturally (apoptosis).

\section{THE TUMOUR GROWTH ALGORITHM}

The tumour growth algorithm follows the steps listed below:

1. Load a two dimensional lattice with a grid size of $\mathrm{N} \times \mathrm{N}$.

2. Prescribe the boundary conditions.

3. Seed five nodes at the centre of the lattice with proliferating cells.

4. Initialise time stepping.

5. Calculate the oxygen concentration level, $C^{t}(x, y)$, at all nodes in the lattice using the finite difference method as described in Solution to the Diffusion Equation.

6. If at a node in the lattice, the cell is in the proliferating state and, if $C^{t}(x, y) \geq k_{p}$, cell division occurs as described in (Automaton Rules 2) above.

7. If at a node, $k_{q} \leq C^{t}(x, y)<k_{p}$, change the state of the cell at that node to the quiescent state.

8. If at a node, $C^{t}(x, y)<k_{q}$, change the state of the cell at that node to the necrotic state, i.e., both proliferating and quiescent cells will be dead.

9. Repeat $6-8$ for 10 time steps. 
10. After 10 time steps, if dead cells are present in the tumour mass, compute the average radius of the necrotic core.

11. Since dead cells do not consume oxygen, calculate the new distribution of oxygen concentration, $C^{t}(x, y)$, at all nodes in the lattice starting from the edge of the tumour where it meets the healthy tissue to the boundary of the necrotic core.

12. Repeat 6 - 8 for the next 10 time steps. In addition, if at a node, $C^{t}(x, y) \geq k_{p}$, and if the cell at that node in the lattice is in the quiescent state, change the state of the cell to the proliferating state.

13. Repeat 10 - 12 and proceed until the edge of grid is reached.

\section{RESULTS AND DISCUSSION}

\subsection{Comparison with tumour spheroid cultures}

We performed 24 growth simulations with time-independent oxygen distribution for both homogeneous and heterogeneous tumours on a lattice growing from $3 \times 3$ cells to $2000 \times$ 2000 cells. The maximum thickness of the proliferating rim obtained averaged at 79 cells in a mean tumour diameter of 322 cells (Fig.5.a). The rim thickened as the tumour grew from a diameter of about 3 cells to about 322 cells. Thereafter, the thickness reduced sharply at first and then kept fluctuating about an average of 10 cells when the diameter exceeded 640 cells on average. This agrees well with the experimental data on Fibroblasts cultures in [38]. The maximum thickness in their spheroid cultures was obtained between 15 - 21 percent of the total growth period. The maximum rim thickness in our simulation occurred at approximately 14 percent of the total growth period. Although the rim thickened to a maximum a little early during the growth period in our time-independent model, we attribute this to the exclusion of glucose in our model. Glucose supports proliferation when the concentration of oxygen is poor [37].

\subsection{Time-independent vs. time-dependent oxygen diffusion solutions}

The amount of oxygen available to the viable cells was above proliferative threshold and therefore, was enough for cells to survive and reproduce in both time-independent (steadystate) and time-dependent cases (Fig.5.b). The maximum difference in the concentration at the boundary of the necrotic core between the time-independent and time-dependent solutions 
was 13 percent. So, the core could be between 6 - 11 cells thinner when predicted using the time-independent model (Eq.4) as compared to the time-dependent model (Eq.2). The thickness of the quiescent layer may be affected to a similar magnitude. However, since only the proliferating cells are responsible for the change in size (volume and diameter) of tumour, the size would remain the same irrespective of the use of time-independent or time-dependent equation.

\section{Possible location of Figure 5.}

Fig.5. (a) Change in the thickness of the proliferating rim in a growing tumour. (b) Complete oxygen profile for $2000 \times 2000$ lattice.

\subsection{Homogeneous vs. heterogeneous growth}

In the case of homogeneous/classical growth, clear symmetrical layers of proliferating, quiescent and necrotic cells were visible, Fig.6.a. In the case of heterogeneous growth, the shape of tumour was asymmetric and the boundaries of the three layers were irregular (Fig.6.b - f). This is corroborated by the fact that in heterogeneous tumour, mutated cancer cells contribute to different velocities of growth of cells in different directions because of dissimilar proliferation and nutrient consumption rates by the various phenotypes.

In our simulations, tumour remains heterogeneous throughout its growth (Fig.6.b - i, and Fig.7) in contrast to the classical model $[7,13]$ where the absence of mutated phenotypes in homogeneous tumour causes it to grow at the same rate in all directions making the tumour spherical (Fig. 6.a).

\section{Possible location of Figure 6.}

Fig.6. (a) Homogeneous/classical tumour growth gives rise to spherical shape. (b-f) Heterogeneous tumour growth: (b) Only proliferating cells are visible in the initial growth periods, (c) quiescent layer begins to form, (d) the size of proliferating rim starts to decrease, (e) formation of necrotic core, (f) maximum size of tumour with its boundaries intact, (g) boundary begins to rupture, (h) progression of rupture and, (i) diminished number of proliferating cells at the tumour boundary. (This figure should be viewed in colour) 


\section{Possible location of Figure 7.}

Fig.7. The number of cells of phenotypes II, III \& IV in tumour volume shows tumour heterogeneity.

\subsection{Circularity}

To measure the deviation of tumour contour from a perfect circle, we calculated its shape factor by using the well-known circularity formula, circularity $=(4 \pi A) / P^{2}$, where, $\mathrm{A}$ is the area of the tumour and $\mathrm{P}$ is the perimeter of the tumour contour. A perfect circle has a circularity of 1 whereas very irregular shapes have circularity close to zero. The circularity for the full-grown tumour in our simulations was measured to be in the range of 0.53 and 0.67. To verify the circularity of tumour shapes so calculated, we plotted the signature of the tumour contours as is commonly done in digital image processing. Fig.8.a shows the normalised variation of the contour of the full-grown tumour Fig.8.b from its centroid. The dashed line represents the radius of the perfect circle with an area equal to that of the tumour.

\section{Possible location of Figure 8.}

Fig.8. (a) Plot of the signature of the tumour contour shown in Fig.8 (b). The angles are measured from the horizontal counter-clockwise.

\subsection{Growth progression}

During the growth, only proliferating cancer cells were visible until the tumour reached a diameter of about 86 cells (Fig.6.b). Thereafter, cells were seen to become quiescent forming a blue layer (Fig.6.c) and the necrotic core was visible only after the diameter exceeded 254 
cells (Fig.6.e). The thickness of the proliferating rim increases initially and then gradually thins down as growth progresses. We observed that when the tumour diameter reached above 1760 cells (Fig.6.f) (approximately $18 \mathrm{~mm}$ ) on average, intercellular adhesion did not support further growth of the avascular tumour. This phenomenon is similar to the findings in [26, 27] where avascular growth is found to be thwarted between a diameter of $1-2 \mathrm{~mm}$ for generic cancer. Although the size of tumour that was permissible for growth was about one order of magnitude bigger in our case, we attribute this to the higher diffusivity of oxygen in soft tissues, in this case, the brain tissue. On further growth simulation, the proliferating rim started to rupture (Fig.6.g). The rupture was seen to creep into the quiescent layer (Fig.6.h) and on progression, severely diminished the size of the proliferating rim creating a disjointed boundary (Fig.6.i). This is explained by the fact that the inter-cellular adhesion between tumour cells does not permit the proliferating cells to grow further at this stage. It is also worth noting here that for further progression, other than initiating angiogenesis, tumour cells begin to lose inter-cellular adhesion subsequently leading it to the secondary growth stage.

Our model predicts that intercellular adhesion plays an important role in thwarting further growth of an avascular tumour after reaching its diffusion-limited size as experimentally established in [3]. The growth curve (Fig.9.a) is a Gompertzian curve with three distinct phases: an initial exponential growth, followed by a linear growth, and finally the beginning of plateau as seen in experiments by Freyer [38]. In their earlier work [39], the growth in spheroid culture was found to be linear for sizes between $200 \mu \mathrm{m}$ and $1600 \mu \mathrm{m}$ in diameter compared to between $440 \mu \mathrm{m}$ and about $1500 \mu \mathrm{m}$ on average in our steady-state model. The maximum number of cells in a completely grown avascular tumour was about 271,466 cells whereas the minimum was about 218,948 cells with a standard deviation of 17,745 cells that accounts for approximately $6.9 \%$ fluctuation from the mean (Table II) and shows that the simulations were modestly consistent. The cell doubling time for the growing tumour increases at a uniform rate (Fig.9.b) similar to what is seen for a growth of tumour with necrosis in [38].

Table II. Average number of cells in the tumour volume in 24 growth simulations

\begin{tabular}{ccccc}
\hline & Maximum & Minimum & Mean & Standard deviation \\
\hline Tumour volume & 271,466 & 218,948 & 257,791 & 17,745 \\
(number of cells) & & & & \\
\hline
\end{tabular}




\section{Possible location of Figure 9.}

Fig.9. (a) Tumour growth follows the Gompertz curve. (b) Cell doubling time increases uniformly for a growing tumour.

\subsection{Quantitative verification}

To quantitatively evaluate the appropriateness of methods proposed in this paper, we need to relate our results to quantitative results of experiments. These are very hard to find because usually papers presenting data on tumour growth such as [30, 37, 38] do not report information necessary to choose parameters for our model. Here we compare the two basic parameters - Volume Doubling Time $\left(T_{d}\right)$ and Cell Loss Factor $(\varphi)$ - that define the growth of any tumour [40] with data from [41], the results from which are also used most recently in [42]. To compute $T_{d}$ and $\varphi$, we have to determine the Cell Cycle Time $\left(T_{c}\right)$ and the Growth Fraction (GF). Cell Cycle Time $\left(T_{c}\right)$ is the period of time required for a proliferating cell to progress from one mitotic division to the next. We use the Cell Cycle Time $\left(T_{c}\right)$ provided in [41]. Growth Fraction (GF) is the ratio of the proliferating cell population to the total tumour cell population. Both the proliferating cell population and the total tumour population are obtained from our CA growth model. $T_{c}$ and GF are then used to calculate the Potential Doubling Time $T_{\text {pot }}$. The expected doubling time in the absence of cell loss is termed the Potential Doubling Time and is given by Eq.6 [41].

$$
T_{\text {pot }}=T_{c} / G F
$$

The Volume Doubling Time $\left(T_{d}\right)$, which is the time interval required for the whole cell population to double in number, is computed using Eq.7.

$$
T_{d}=\Delta t \log 2 / \log \left(N_{t}-N_{0}\right)
$$

where, $N_{0}$ is the initial number of cells in the tumour volume and $N_{t}$ is the number of cells after time $\Delta t$. The Volume Doubling Time $\left(T_{d}\right)$ will be equal to the Cell Cycle Time $\left(T_{c}\right)$ when all cells in the tumour volume are proliferating. 
We then compute the Cell Loss Factor $(\varphi)$. The Cell Loss Factor represents the rate of cell loss as a fraction of the rate at which cells are added to the total population by mitosis and is computed using Eq.8 [41].

$$
\varphi=1-\left(T_{\text {pot }} / T_{d}\right)
$$

$T_{p o t}$ and $T_{d}$ in Eq.8 are obtained from Eq.6 and Eq.7 respectively.

Here, we compare results from our CA model with the findings on 4-day, 7-day and 13day experimental tumour Ehrlich ascites carcinoma [41] \& references therein. Since the Cell Cycle Time $\left(T_{c}\right)$ is constant in our CA model, we also choose experimental data set pertaining to a constant $T_{c}$. The post-synthesis (premitotic) gap phase $\mathrm{G}_{2}=6 \mathrm{hrs}$ is chosen to be equivalent to $T_{c}$ in the experiment as synthesis is not considered in our model. For consistency, we use and present all measurements of time in units of $T_{c}$.

4-day Ehrlich ascites: To compare the 4-day Ehrlich ascites with our growth model, we first choose the tumour at a growth stage in our model that has the same value of Cell Loss Factor $(\varphi)$ as that for the 4-day Ehrlich ascite in the experiment (see Table III). Since $\varphi=0$ in this case, no cells are lost during the growth process. Therefore, the Growth Factor is 1. The experimentally observed $T_{d}$ in this case is $3.4 T_{c}$. The value of $T_{d}$ obtained from our CA model that is calculated using Eq.7 is $3.1 T_{c}$.

7-day Ehrlich ascites: To compare the 7-day Ehrlich ascites, we choose the size of tumour from our CA model that has the Cell Loss Factor $(\varphi)$ closest to that observed in the experiment. The closest match to the experimental $\varphi=42.0 \%$ is $45.8 \%$ in our model (Table III). To proceed to find $T_{d}$, we first calculate the growth fraction, $G F=P /(P+Q)$. $\mathrm{P}$ and $\mathrm{Q}$ are the number of proliferating and quiescent cells respectively and are obtained from our CA model. GF is used to calculate the Potential Doubling Time $\left(T_{\text {pot }}\right)$ using Eq.6. The Volume Doubling Time $\left(T_{d}\right)$ is then obtained for our CA model by using Eq.7. The value of $T_{d}$ thus obtained for our CA model is higher than that of the experiment. However, the Cell Loss Factor $(\varphi)$ for our CA model was also higher than that of the experiment. A higher Cell Loss Factor implies that a higher fraction of cells is lost from the tumour volume and therefore, the Volume Doubling Time also becomes higher. However, the large difference between the Volume Doubling Times could not be explained.

13-day Ehrlich ascites: We proceed to compare the 13-day Ehrlich ascites measurements to those of our CA model in a method similar to that followed for 7-day Ehrlich ascites. In 
this case, the closest match to the experimental $\varphi=89.0 \%$ is $81.3 \%$ in our model. The Volume Doubling Time $\left(T_{d}\right)$ calculated for our model is smaller than that of the experiment. Since the Cell Loss Factor $(\varphi)$ for our model is also smaller than that of the experiment, a smaller fraction of cells is lost from the tumour volume in the CA model compared to the experiment. Therefore, the Volume Doubling Time is smaller for the CA model.

Table III: Parametric comparison between experiment and CA model

\begin{tabular}{|c|c|c|c|c|c|}
\hline \multirow[b]{2}{*}{ Tumour } & \multirow[b]{2}{*}{$\begin{array}{c}\text { Cell Cycle } \\
\text { Time } \\
\mathrm{T}_{\mathrm{c}}(\mathrm{hrs})\end{array}$} & \multicolumn{2}{|c|}{$\begin{array}{c}\text { Cell Loss Factor } \\
\varphi(\%)\end{array}$} & \multicolumn{2}{|c|}{$\begin{array}{l}\text { Volume Doubling Time } \\
T_{d}\left(\text { in units of } T_{c}\right)\end{array}$} \\
\hline & & Experiment & CA Model & Experiment & CA Model \\
\hline \multicolumn{6}{|l|}{$\begin{array}{l}\text { Ehrlich } \\
\text { ascites }\end{array}$} \\
\hline $\begin{array}{r}\text { 4-day } \\
\text { tumour }\end{array}$ & 6.0 & 0.0 & 0.0 & 3.4 & 3.1 \\
\hline $\begin{array}{l}\text { 7-day } \\
\text { tumour }\end{array}$ & 6.0 & 42.0 & 45.8 & 6.4 & 11.3 \\
\hline $\begin{array}{l}\text { 13-day } \\
\text { tumour }\end{array}$ & 6.0 & 89.0 & 81.3 & 56.0 & 43.1 \\
\hline
\end{tabular}

Other sources of error may be the difficulties associated with the measurements of viable cells in the experimental tumours. In [41], it is assumed that the thymidine-labelling index indicates the proportion of cells although the measurement is affected by the finite (short) availability time of tritiated thymidine. It is also assumed that thymidine reaches all cells after injection. The occurrence of 'false negatives' will lead to an overestimate of Potential Doubling Time and an under-estimate of cell loss. The results, however, follow the general observation that an increase in tumour size is accompanied by greater cell loss, a lower growth fraction, and a longer doubling time [40].

\section{CONCLUSIONS}

We conclude that our CA model incorporating heterogeneous cell population is able to capture the tumour growth dynamics at the cellular level that is in good agreement with existing experimental data. The choice of time-independent (steady-state) solution to the distribution of oxygen in the tumour volume allows faster implementation of the model 
without losing the characteristics of a time-dependent solution. Other nutrients such as glucose can be easily introduced into the model by including its diffusivity. Further, the model can also be used to study the effect of treatment by incorporating the change of state of cells upon exposure to a threshold concentration of radiation.

We are currently in the process of extending the model to three dimensions, and also studying the growth dynamics by including other factors such as the effect of surrounding normal tissue. Earlier work by our group shows that the speed and efficiency of computation of brain deformations is significantly increased through implementation on GPU [43]. However, implementation of our 3D tumour model in GPU presents challenges due to the insufficient internal memory of current GPUs that are not capable of holding the complete data during the simulation of the $3 \mathrm{D}$ growth model.

Another challenge lies in verifying the model with a wide range of clinical data. Our clinical collaborators suggest that clinicians currently cannot provide data that could be directly applicable for direct, quantitative verification of our methods as the resolution of imaging equipment used for diagnostics is too crude. It may be added here that data from clinical trials on glioma growth exist. However, none of it has been released publicly, as far as we know, and often this type of very valuable data is the property of pharmaceutical companies funding the trial.

The best way to verify our method would be to inject tumour cells stereotactically into mice Caudate Nuclei, which is a standard method for tumour-growth and subsequent therapeutic trials. The growth of these lesions can be monitored by $7 \mathrm{~T}$ MRI and provides the purest data to validate our methods. Also oxygen and nutrients could be directly measured with other imaging methods. We are planning, with our clinical collaborators, to embark on such an experiment.

\section{Acknowledgements}

The first author was a SIRF scholar in Australia and was in receipt of the UIS scholarship during the completion of this research. The financial support of the National Health and Medical Research Council (Australia) Grant No.1006031 and the Research Collaboration Award 2011 at the University of Western Australia are gratefully acknowledged. 


\section{References}

1. Wolfgang A. Molecular biology of human cancers. Springer, Netherlands, 2007

2. Hanahan D. The hallmarks of cancer. Cell 2000; 100(1):57.

3. Sutherland R. Cell and environment interactions in tumor microregions: The multicell spheroid model. Science 1988; 240(4849):177-184.

4. Qi A-S, Zheng X, Du C-Y,An B-S. A cellular automaton model of cancerous growth. Journal of Theoretical Biology 1993; 161(1):1-12.

5. Dormann S. Modeling of self-organized avascular tumor growth with a hybrid cellular automaton. In silico biology 2002; 2(3):393.

6. Vermeulen L. Single-cell cloning of colon cancer stem cells reveals a multi-lineage differentiation capacity. Proceedings of the National Academy of Sciences of the United States of America 2008; 105(36):13427.

7. Greenspan H. Models for the growth of a solid tumour by diffusion. Studies in Applied Mathematics 1972; 4:317-340.

8. Gatenby RA. A reaction-diffusion model of cancer invasion. Cancer Research 1996; 56(24):5745.

9. Ward JP. Mathematical modelling of avascular-tumour growth. Mathematical medicine and biology 1997; 14(1):39.

10. Ferreira Jr SC,Ferreira. Reaction-diffusion model for the growth of avascular tumor. Physical review. E, Statistical physics, plasmas, fluids, and related interdisciplinary topics 2002; 65(2):021907.

11. Ambrosi D. On the mechanics of a growing tumor. International journal of engineering science 2002; 40(12):1297.

12. Byrne H. Modelling solid tumour growth using the theory of mixtures. Mathematical medicine and biology 2003; 20(4):341.

13. Cristini V. Nonlinear simulations of solid tumor growth using a mixture model: Invasion and branching. Journal of mathematical biology 2009; 58(4-5):723.

14. Araujo RP,Araujo. A history of the study of solid tumour growth: The contribution of mathematical modelling. Bulletin of mathematical biology 2004; 66(5):1039.

15. Wang Z, Yip S,Diaz de la Rubia T. Computational modeling of brain tumors: Discrete, continuum or hybrid? Scientific modeling and simulations. Vol. 68, 2008

16. Anderson ARA. A hybrid mathematical model of solid tumour invasion: The importance of cell adhesion. Mathematical medicine and biology 2005; 22(2):163.

17. Alarcon T. Towards whole-organ modelling of tumour growth. Progress in biophysics and molecular biology 2004; 85(2-3):451.

18. Sottoriva A, Verhoeff JJC, Borovski T, McWeeney SK, Naumov L, Medema JP, Sloot PMA,Vermeulen L. Cancer stem cell tumor model reveals invasive morphology and increased phenotypical heterogeneity. Cancer Research 2010; 70(1):46-56.

19. Chen Y. Design optimization of scaffold microstructures using wall shear stress criterion towards regulated flow-induced erosion. Journal of biomechanical engineering 2011; 133(8):081008.

20. $\mathrm{Li}$ WEI. Finite element based bone remodeling and resonance frequency analysis for osseointegration assessment of dental implants. Finite elements in analysis and design 2011; 47(8):898.

21. Wolfram S. Theory and applications of cellular automata. World Scientific: Singapore,

1986 
22. Wolfram S. New kind of science. Wolfram Media, Champaign, IL, 2002

23. Kansal AR, Torquato S, Chiocca EA,Deisboeck TS. Emergence of a subpopulation in a computational model of tumor growth. Journal of Theoretical Biology 2000; 207(3):431-441.

24. Gevertz JL. Modeling the effects of vasculature evolution on early brain tumor growth. Journal of Theoretical Biology 2006; 243(4):517.

25. Gerlee P. An evolutionary hybrid cellular automaton model of solid tumour growth. Journal of Theoretical Biology 2007; 246(4):583.

26. Wise SM. Three-dimensional multispecies nonlinear tumor growth--i:: Model and numerical method. Journal of Theoretical Biology 2008; 253(3):524.

27. Frieboes HB. Three-dimensional multispecies nonlinear tumor growth-ii: Tumor invasion and angiogenesis. Journal of Theoretical Biology 2010; 264(4):1254.

28. Shrestha S, Joldes G, Wittek A,Miller K, Modeling heterogeneous tumor growth using hybrid cellular automata, in Computational biomechanics for medicine, P.M.F. Nielsen, A. Wittek, and K. Miller, Editors. 2012, Springer New York. 129-139.

29. Sherwood L. Human physiology: From cells to systems. Brooks/Cole, 2010

30. Tomita K. In vivo cell cycle synchronization of the murine sarcoma 180 tumor following alternating periods of hydroxyurea blockade and release. Cancer Research 1979; 39(11):4407.

31. Casciari JJ. Variations in tumor cell growth rates and metabolism with oxygen concentration, glucose concentration, and extracellular ph. Journal of cellular physiology 1992; 151(2):386.

32. Belytschko T, Hughes, TJR. Computational methods for transient analysis. NorthHolland, Amsterdam, 1983

33. Joldes GR. An adaptive dynamic relaxation method for solving nonlinear finite element problems. Application to brain shift estimation. International journal for numerical methods in biomedical engineering 2011; 27(2):173.

34. Joldes GR. Stable time step estimates for mesh-free particle methods. International journal for numerical methods in engineering 2012; 91(4):450.

35. Berger J, Horton A, Joldes G, Wittek A,Miller K. Coupling finite elements and meshfree methods for modelling brain deformation in response to tumour growth. Computational Biomechanics for Medicine 2008(1):1-14.

36. Gerlee P,Anderson ARA. Stability analysis of a hybrid cellular automaton model of cell colony growth. Physical Review E 2007; 75(5):051911.

37. Freyer JP. In situ oxygen consumption rates of cells in v-79 multicellular spheroids during growth. Journal of cellular physiology 1984; 118(1):53.

38. Freyer JP. Role of necrosis in regulating the growth saturation of multicellular spheroids. Cancer Research 1988; 48(9):2432.

39. Landry J. A model for the growth of multicellular spheroids. Cell Proliferation 1982; 15(6):585.

40. Hoshino T,Wilson CB. Review of basic concepts of cell kinetics as applied to brain tumors. Journal of Neurosurgery 1975; 42(2):123-131.

41. Steel GG. Cell loss from experimental tumours. Cell Proliferation 1968; 1(3):193207.

42. Talmadge JE. Murine models to evaluate novel and conventional therapeutic strategies for cancer. The American journal of pathology 2007; 170(3):793.

43. Joldes GR. Real-time nonlinear finite element computations on gpu-application to neurosurgical simulation. Computer methods in applied mechanics and engineering 2010; 199(49-52):3305. 


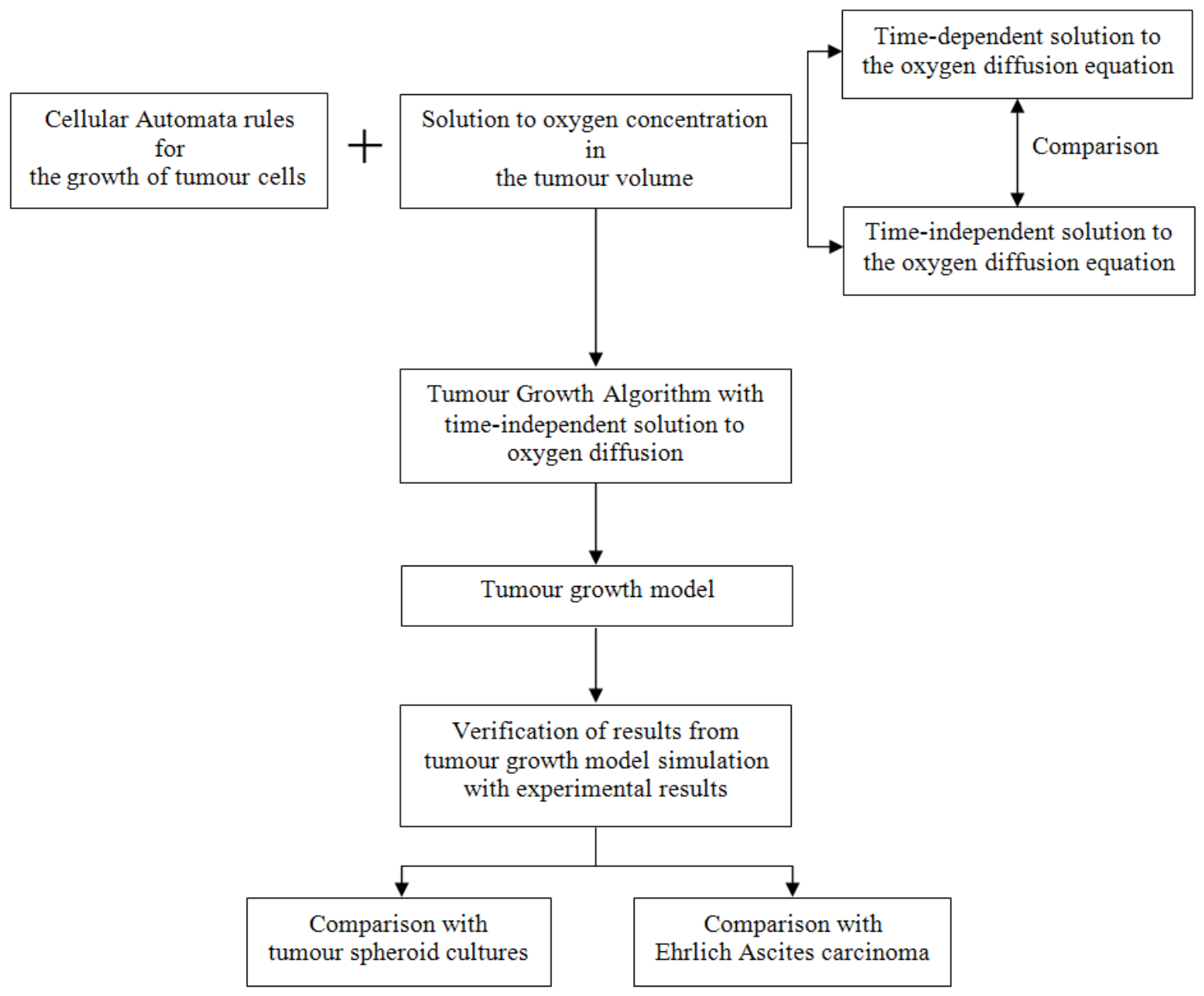

Fig.1. The scheme of study 


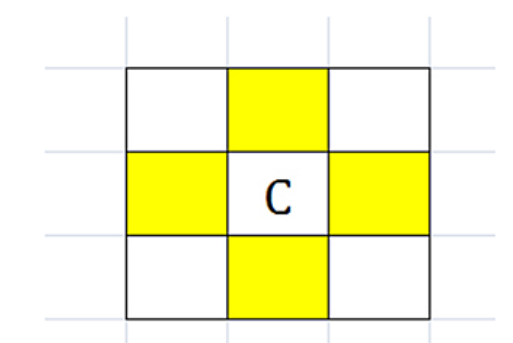

a)

Fig.2. (a) The von Neumann neighbourhood consists of cells at positions $(0,0),( \pm 1,0),(0, \pm 1)$, i.e., the four yellow cells and the cell C. (b) A daughter cell can take position at site 1, 2, 3 or 4 with equal probability. However, the cell existing at that site has to be displaced to one of its neighbouring blue sites first to create space for the daughter cell. 


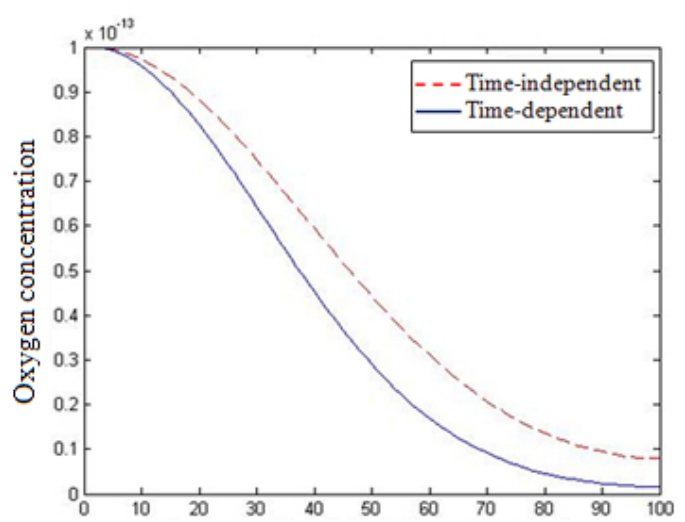

a)

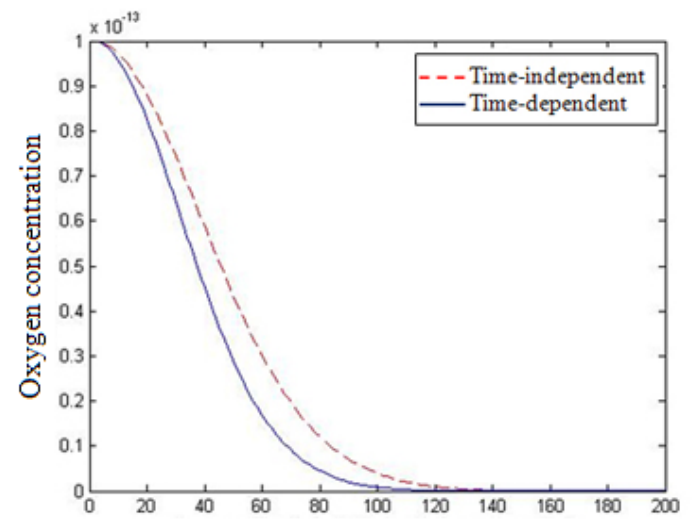

c)

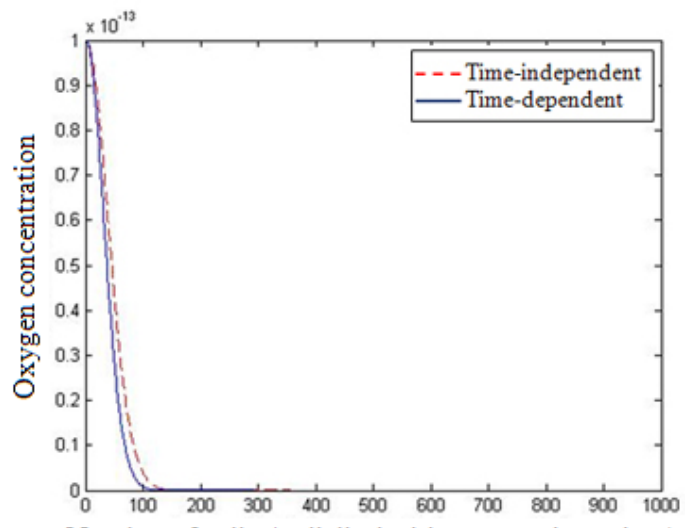

e) Number of cells (radially inside tumour boundary)

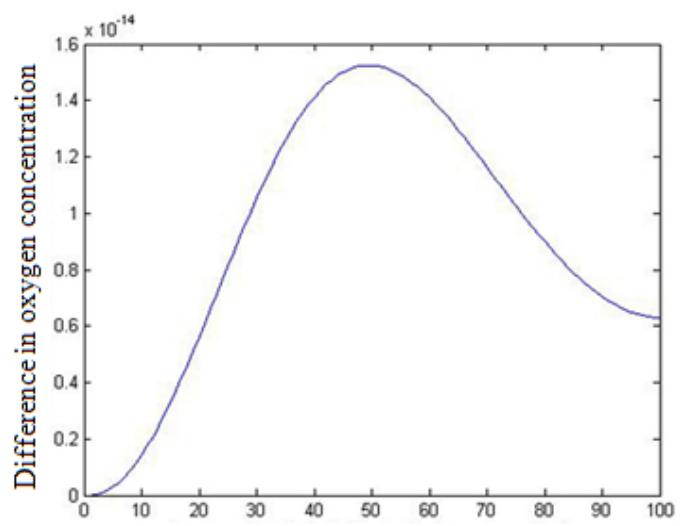

b)

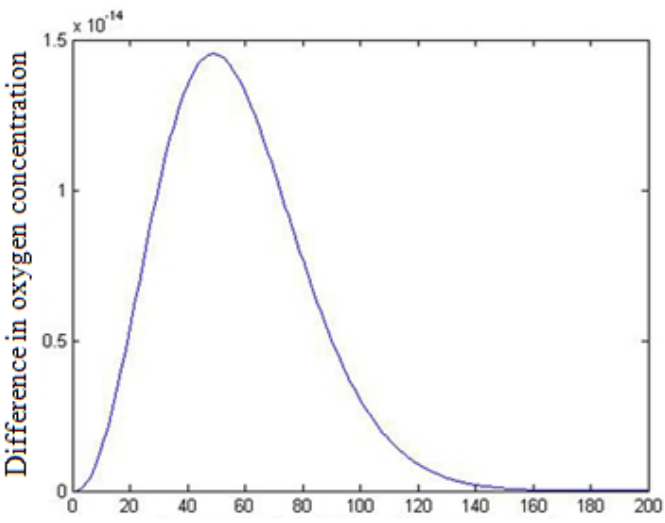

d)

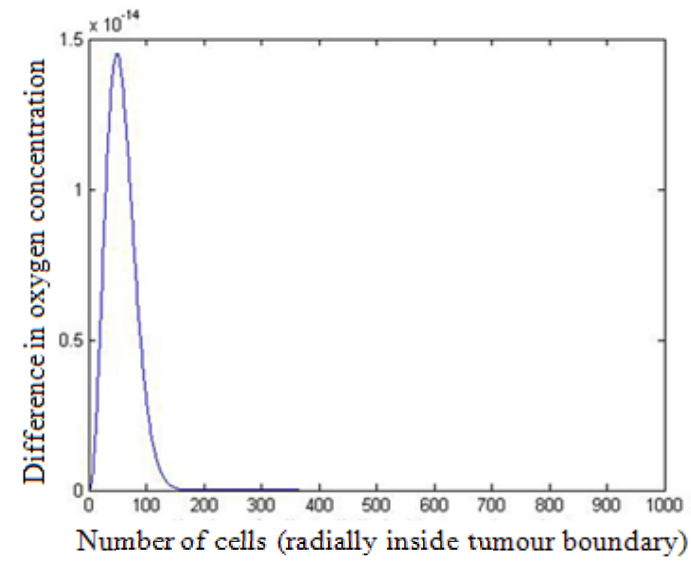

Fig.3. (a), (c), (e) Oxygen profiles (concentrations in g cell $^{-1}$ ) using steady-state timeindependent (Laplace) and time-dependent equations for fixed lattice sizes of $200 \times 200$, $400 \times 400$, and $2000 \times 2000$ cells respectively. Profiles are shown from the tumour boundary to its centre. (b), (d), (f) The difference in oxygen concentration corresponding to the three lattice sizes respectively. 
a)

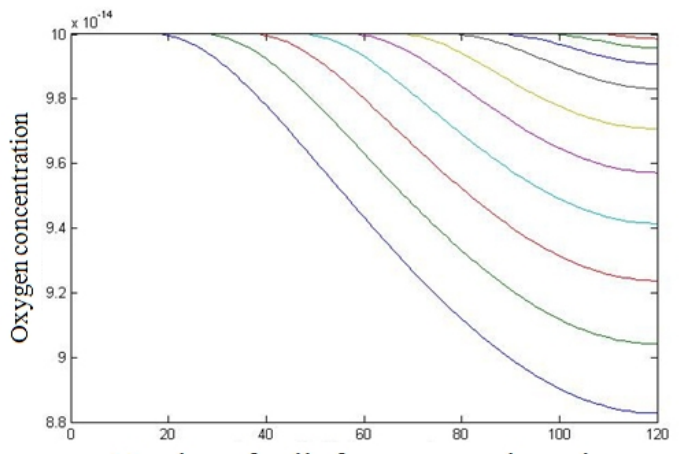
Number of cells from tumour boundary

c)

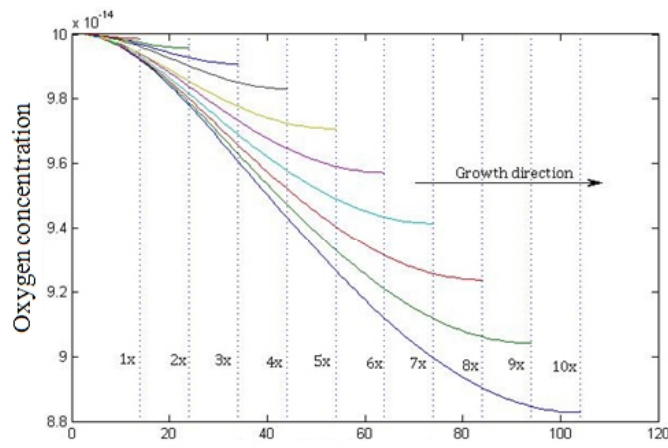

Number of cells from tumour boundary

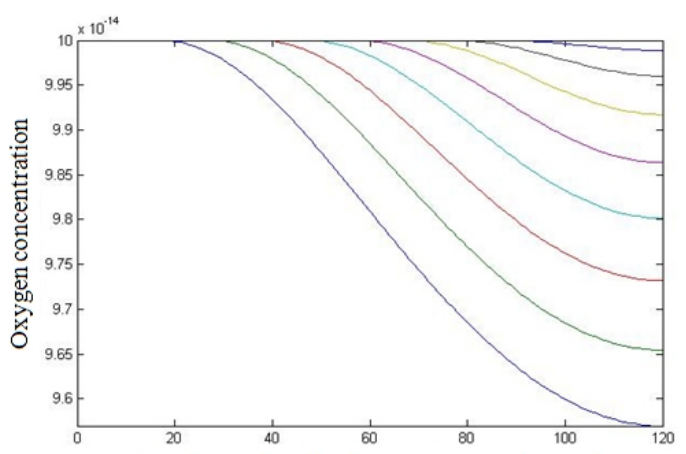

b)

Number of cells from tumour boundary

d)

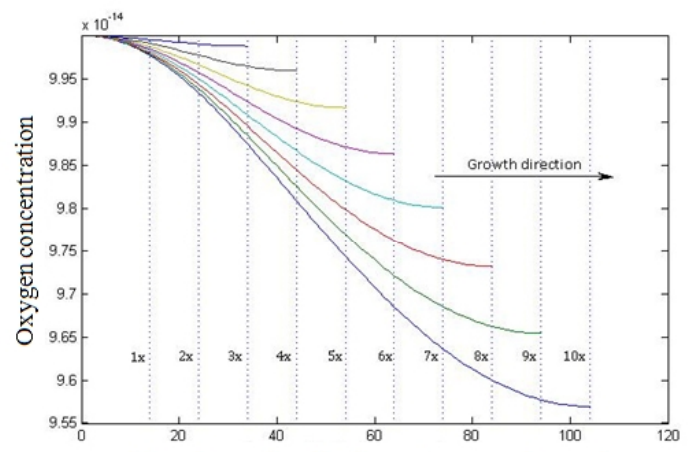

Number of cells from tumour boundary

e)

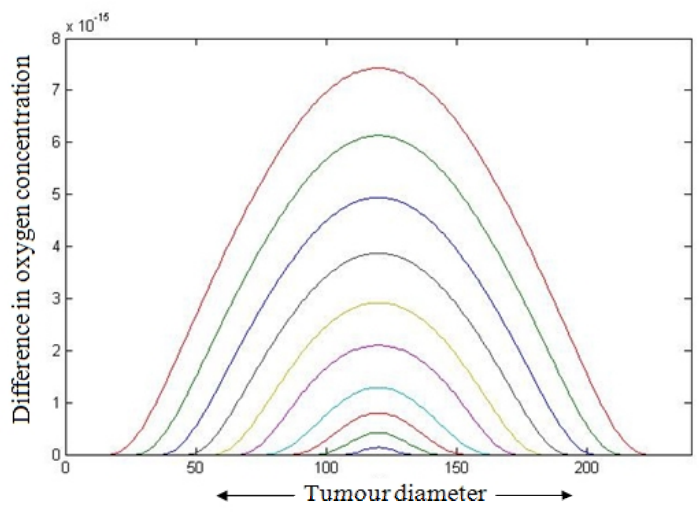

Fig.4. Oxygen profiles (concentrations in g cell $^{-1}$ ) using (a) time-independent and (b) time-dependent diffusion equations for varying lattice sizes. The right edge of each plot (120 cells) represents the centre of tumour volume. Profiles at various blocks of time with their corresponding sizes of lattice using (c) time-independent and (d) time-dependent equations for varying lattice sizes. The left edge of each plot represents the boundary of the tumour volume. (e) Difference in the oxygen concentration between the steady-state time-independent and time-dependent solutions for different tumour diameters (in number of cells). 


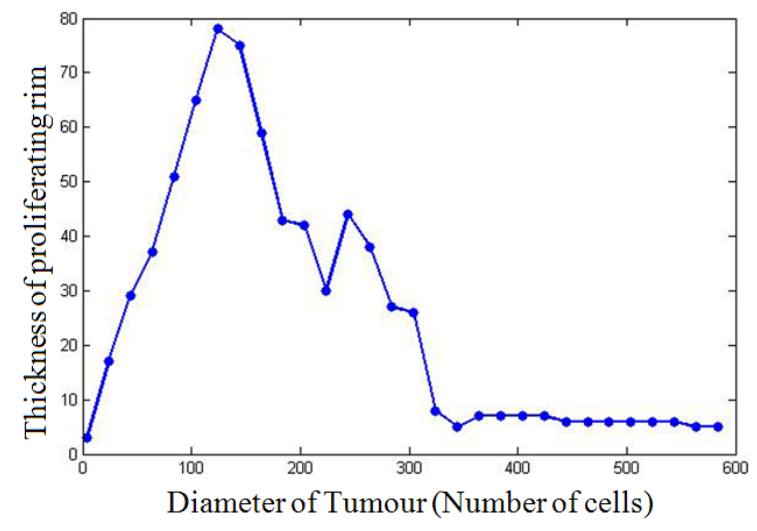

a)

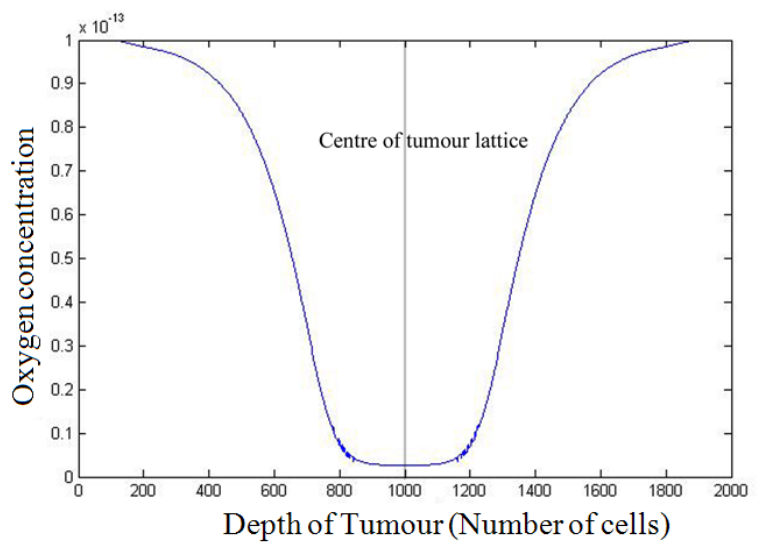

b)

Fig.5. (a) Change in the thickness of the proliferating rim in a growing tumour. (b) Complete oxygen profile for $2000 \times 2000$ lattice. 

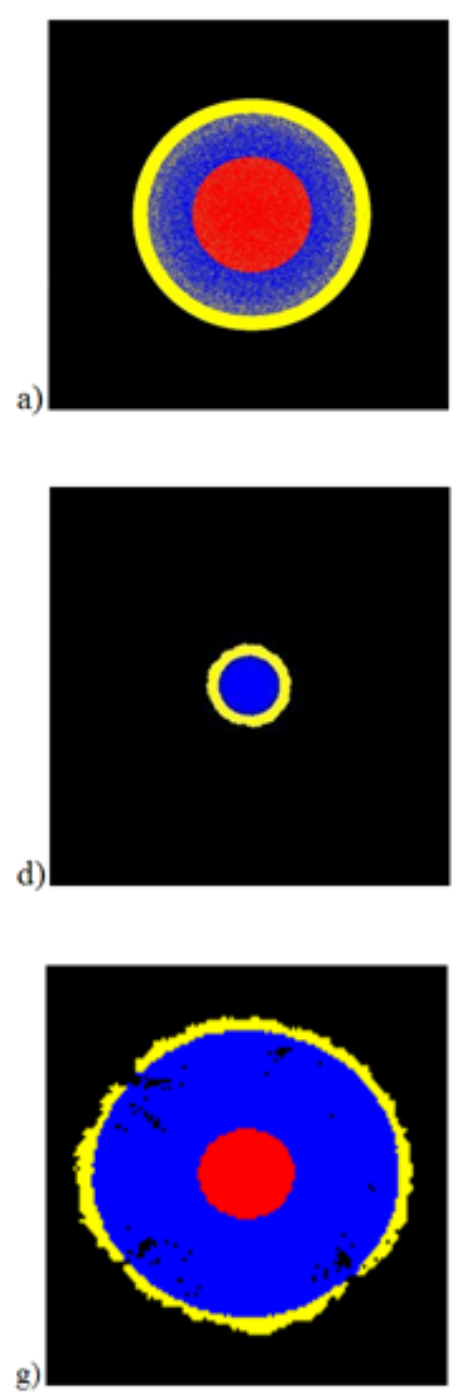

b)
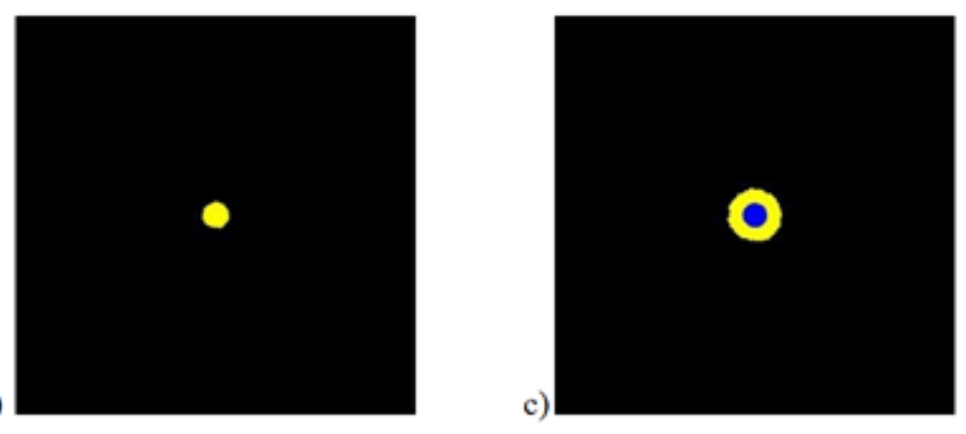

e)

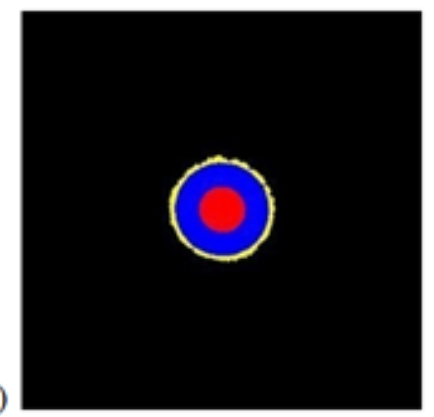

f)

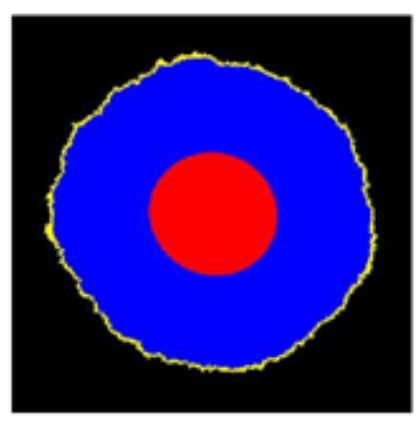

h)
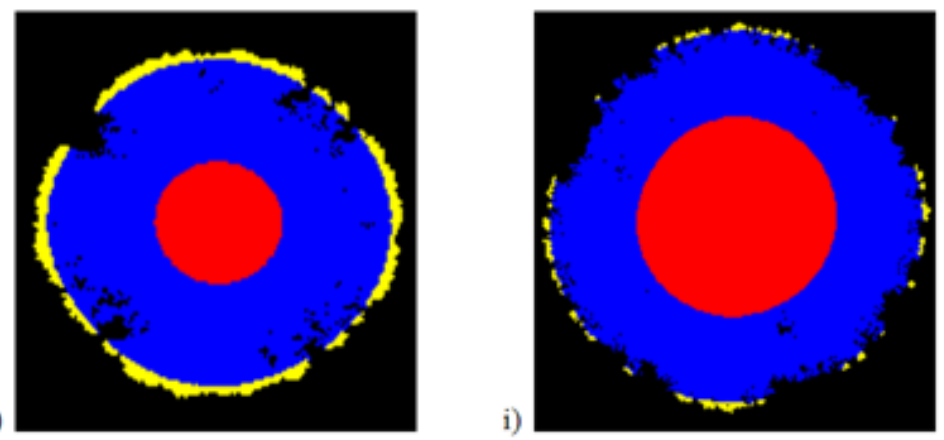

Fig.6. (a) Homogeneous/classical tumour growth gives rise to spherical shape. (b-f) Heterogeneous tumour growth: (b) Only proliferating cells are visible in the initial growth periods, (c) quiescent layer begins to form, (d) the size of proliferating rim starts to decrease, (e) formation of necrotic core, (f) maximum size of tumour with its boundaries intact, (g) boundary begins to rupture, (h) progression of rupture and, (i) diminished number of proliferating cells at the tumour boundary. (This figure should be viewed in colour) 


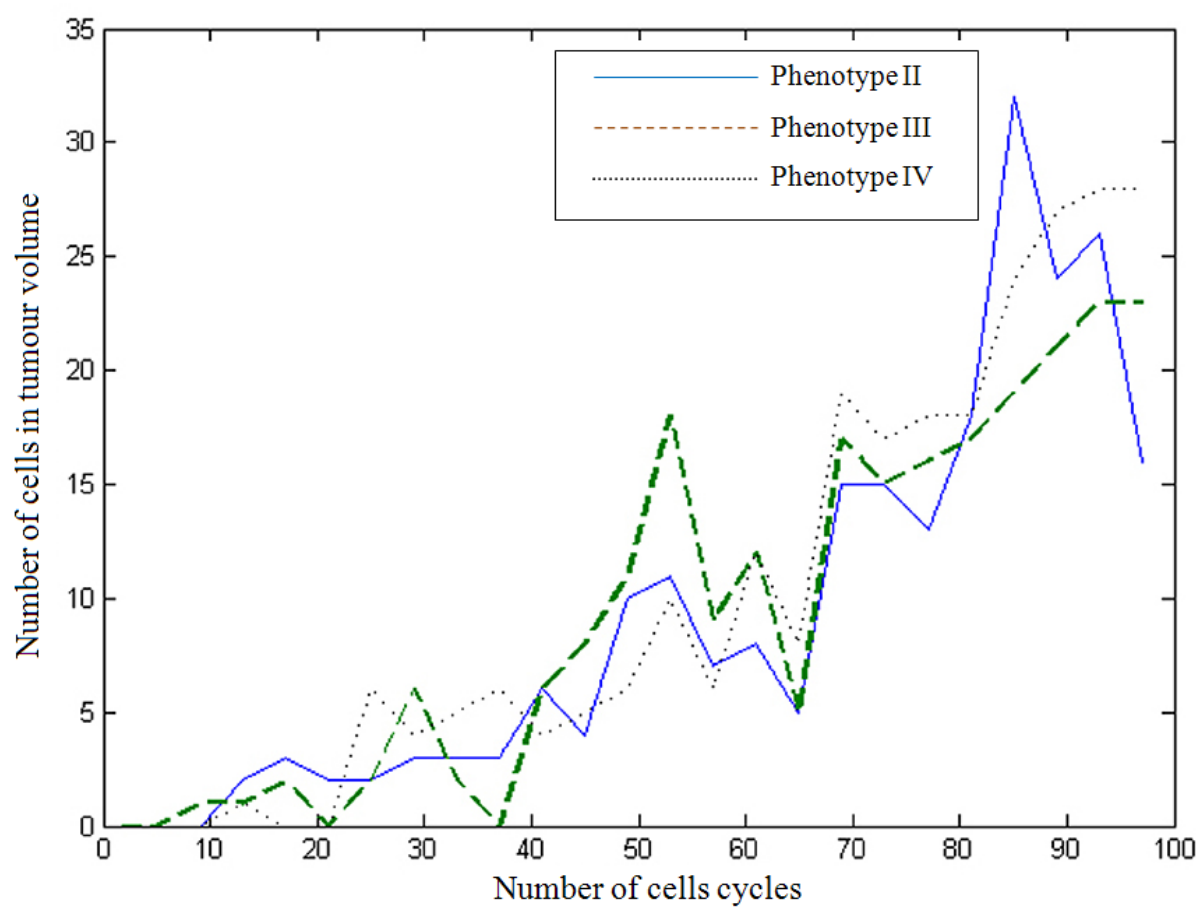

Fig.7. The number of cells of phenotypes II, III \& IV in tumour volume shows tumour heterogeneity. 


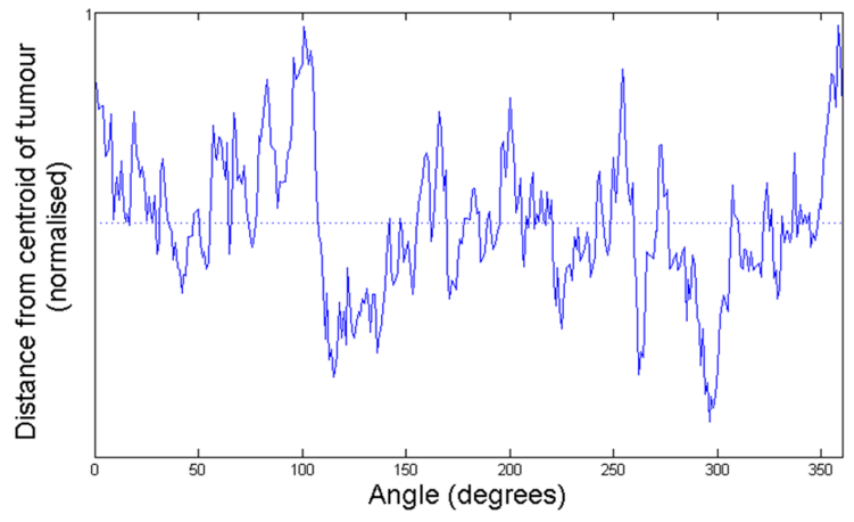

Fig.8. (a) Plot of the signature of the tumour contour shown in Fig.8. (b). The angles are measured from the horizontal counter-clockwise.

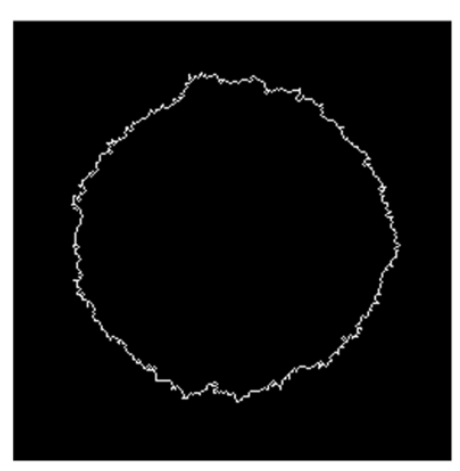

b) 


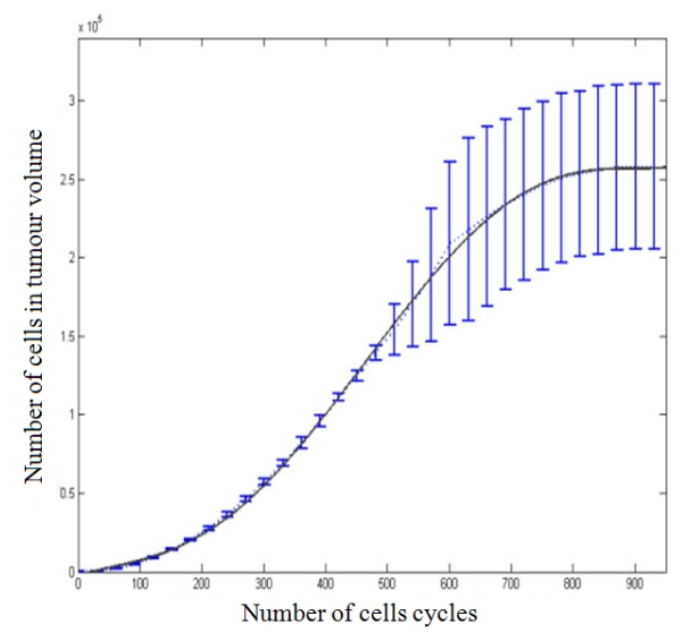

a)

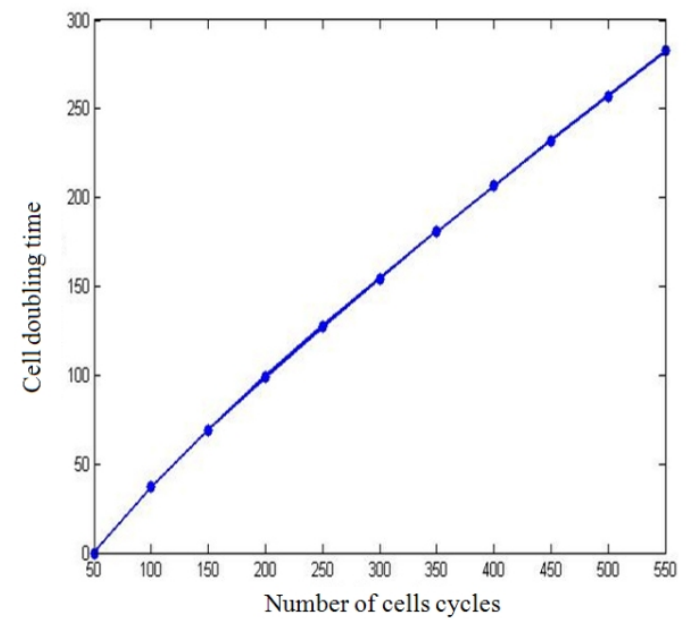

b)

Fig.9. (a) Tumour growth follows the Gompertz curve. (b) Cell doubling time increases uniformly for a growing tumour. 